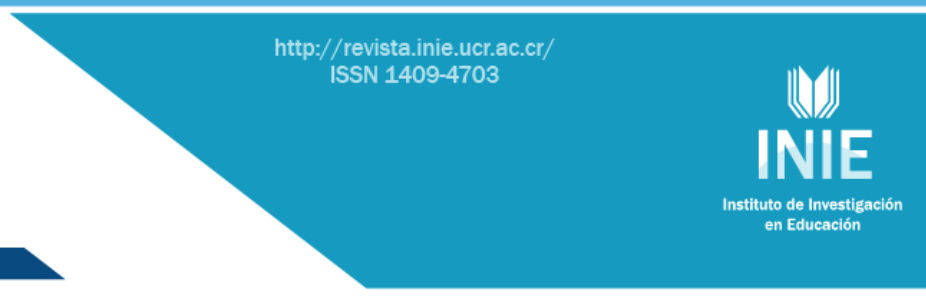

\title{
COMPRENSIÓN LECTORA, MEMORIA DE TRABAJO, FLUIDEZ Y VOCABULARIO EN ESCOLARES CUBANOS
}

READING COMPREHENSION V/S WORKING MEMORY, FLUENCY, AND VOCABULARY IN CUBAN STUDENTS

\section{Volumen 16, Número 1}

Enero - Abril

pp. 1-18

Klency González Hernández

Laura Otero Paz

Ana Maria Castro Laguardia

Revista indizada en REDALYC, $\underline{\text { SCIELO }}$

Revista distribuida en las bases de datos:

LATINDEX, DOAJ, E-REVIST@S, IRESIE, CLASE, DIALNET, SHERPA/ROMEO, QUALIS,

Revista registrada en los directorios:

ULRICH'S, REDIE, RINACE, OEI, MAESTROTECA, PREAL, $\underline{\text { CLACSO }}$ 


\title{
COMPRENSIÓN LECTORA, MEMORIA DE TRABAJO, FLUIDEZ Y VOCABULARIO EN ESCOLARES CUBANOS \\ READING COMPREHENSION VIS WORKING MEMORY, FLUENCY, AND VOCABULARY IN CUBAN STUDENTS
}

\author{
Klency González Hernández ${ }^{1}$ \\ Laura Otero Paz ${ }^{2}$ \\ Ana María Castro Laguardia ${ }^{3}$
}

\begin{abstract}
Resumen: El presente artículo examina algunos factores cognitivos asociados a las dificultades de comprensión lectora de un grupo de estudiantes de tercero y quinto grados del municipio Marianao, provincia La Habana, Cuba en el año 2013. Participaron 108 estudiantes, 54 del sexo femenino y 54 del sexo masculino, con edades comprendidas entre los 8 y 10 años. Fueron evaluadas la comprensión lectora, la memoria de trabajo verbal, la fluidez y el vocabulario. Los principales resultados muestran que la memoria de trabajo verbal, la fluidez y el vocabulario se correlacionan positiva y significativamente con la comprensión lectora, por lo que pueden considerarse buenos predictores del desempeño en relación con este proceso. En relación con cada una de estas variables se observa un mejor rendimiento de los estudiantes de quinto grado, con respecto a los de tercer grado.
\end{abstract}

Palabras clave: COMPRENSIÓN, MEMORIA DE TRABAJO, FLUIDEZ, VOCABULARIO, EDUCACIÓN PRIMARIA, CUBA.

Abstract: Present article examined some factors that can be related to the reading comprehension difficulties that emerge within a group of $3^{\text {rd }}$ and $5^{\text {th }}$ graders from municipality Marianao, in Havana, Cuba in 2013. The sample consisted of 108 students, of which $50 \%$ are female and the other half are males. The ages ranged from 8 to 10 years old. Reading fluency, verbal working memory, vocabulary and reading comprehension were evaluated. Results show that verbal working memory, reading fluency and vocabulary correlate positive and significantly with reading comprehension. Because of that, this factor could be consider as good predictors of the reading comprehension performance. There were observe a better performance related to each variable in students from fifth grade.

Key words: COMPREHENSION, WORKING MEMORY, FLUENCY, VOCABULARY, PRIMARY EDUCATION, CUBA.

\footnotetext{
1 Profesora Auxiliar e investigadora de la Facultad de Psicología de la Universidad de La Habana, Cuba. Máster en Psicología Educativa. Dirección electrónica: klency@psico.uh.cu

${ }^{2}$ Adiestrada de la Facultad de Psicología de la Universidad de La Habana, Cuba. Licenciada en Psicología. Dirección electrónica: lauraotero@psico.uh.cu

3 Adiestrada del Departamento de Neurociencias Cognitivas y Sociales. Centro de Neurociencias de Cuba. Dirección electrónica: anamaria.castro@cneuro.edu.cu
}

Artículo recibido: 24 de febrero, 2015

Enviado a corrección: 24 de junio, 2015

Aprobado: 5 de octubre, 2015 


\section{Introducción}

Saber leer no es solo poder decodificar un conjunto de grafemas y pronunciarlos de manera correcta. Se trata también de comprender aquello que se lee. La comprensión lectora es un proceso cíclico de construcción e integración de información- en el que participan otros procesos cognitivos que permiten formar y conectar proposiciones dentro de una frase, realizar inferencias y elaborar macroideas- para construir una representación coherente del texto en el que se incluye tanto la información extraída como los conocimientos poseídos por el lector. (Kintsch, 1998 y Martínez, Vidal- Abarca, Sellés, y Gilabert, 2008).

Durante el proceso de enseñanza-aprendizaje, desde la primaria hasta la educación postgraduada, se necesita comprender una variada gama de textos para apropiarse de conocimientos. Sin embargo, existe una diferencia entre la enseñanza de la lectura y de la comprensión. Mientras que a la primera se le dedican años de aprendizaje en la escuela, la educación formal pocas veces se ocupa de enseñar la comprensión, por lo que esta se convierte en una demanda para la cual el estudiante muchas veces no cuenta con las habilidades necesarias.

Dada la complejidad de ambos procesos no resulta sorprendente que muchos niños atraviesen por serias dificultades para aprender a leer, y no pocos adultos mantengan en niveles superiores de educación estas dificultades. Estudios a nivel mundial reportan preocupantes índices de prevalencia de las dificultades en comprensión lectora. Según informes de investigación, en Estados Unidos, por ejemplo, el 33\% de alumnos de cuarto grado y el $26 \%$ de alumnos de octavo grado, leen por debajo del nivel básico (Perfetti, Landi, y Oakhill, 2005). Por su parte, estudios realizados en el Reino Unido sugieren que un $10 \%$ de los niños entre los 7 y 11 años de edad muestran problemas para comprender textos (Ricketts, Nation, y Bishop, 2007; Snowling y Hulme, 2011). En Argentina e Italia, el porcentaje de estudiantes con dificultades específicas para la comprensión lectora oscila entre el $5 \%$ y el $10 \%$ de la población escolar total (Abusamra, Ferreres, Raiter, De Beni y Cornoldi, 2010).

Las autoras del presente estudio, no han tenido acceso a investigaciones realizadas en Cuba que develen qué porcentaje de estudiantes presenta dificultades de comprensión, ni cuáles son esas dificultades. Tampoco se han identificado intervenciones que desde una perspectiva cognitiva, en la enseñanza primaria o secundaria, se centren en mejorar este proceso. Solo se ha referenciado un estudio realizado con 90 estudiantes de nuevo ingreso a 
la enseñanza superior a quienes se les aplicó un programa para mejorar la comprensión de textos mediante el uso de estrategias (González, 2008). Los resultados de dicho trabajo apuntan a una mejora de la comprensión lectora de los participantes. Antes de la intervención, solo el 35\% de los estudiantes era capaz de construir una representación proposicional del texto y el 23 \% una representación situacional. Al finalizar la intervención, el $77 \%$ de los lectores pudieron construir la representación proposicional, mientras que el 90\% construyó la representación situacional. La autora resalta la necesidad de realizar estudios de este tipo en grados inferiores de educación- para enseñar a los lectores a comprender textos una vez que hayan aprendido a leer.

Teniendo en cuenta la complejidad del proceso de comprensión, las múltiples variables que pueden afectarlo, los datos sobre la prevalencia de las dificultades de comprensión y la existencia en Cuba de insuficientes estudios de este tipo, la presente investigación, realizada en 2013, se propuso identificar algunos factores asociados a las dificultades de comprensión lectora en escolares cubanos de $3 r o$ y 5 to grados.

\section{Breve referente teórico}

La comprensión constituye la finalidad del acto de leer y puede ser definida como un proceso interactivo y estratégico que implica la construcción de una representación mental del significado del texto, poniendo en relación las ideas contenidas con conocimientos previos almacenados en la Memoria a Largo Plazo (Kintsch y Van Dijk, 1978; McNamara y Magliano, 2009). Estudiar las múltiples variables y procesos que ocurren durante la comprensión de textos ha posibilitado realizar esfuerzos para mejorar el desempeño de los lectores. Muchos son los factores que inciden en las dificultades de comprensión. Diversas investigaciones consultadas (Cain, Bryant y Oakhill, 2004; Carretti, Borella, Cornoldi y De Beni, 2009; Catts, Adlof, y Ellis-Weismer, 2006; Cromley, Snyder-Hogan y Luciw-Dubais, 2014 y Nation, Snowling y Clark, 2007) señalan como algunos de los más importantes la memoria de trabajo verbal, la fluidez lectora y el vocabulario.

La memoria de trabajo se refiere al conjunto de procesos y contenidos activados en forma temporal, involucrados en el control, la regulación y el mantenimiento activo de información relevante para una tarea, al servicio de la cognición compleja (Baddeley y Hitch, 1974). Es un sistema activo de almacenamiento temporario y procesamiento simultáneo y activo de la información que posee una capacidad limitada (Demagistri, Canet, Naveira y Richard, 2012). En un estudio realizado por Swanson y Beebe-Frankenberger (2004), la 
memoria de trabajo verbal resultó ser un buen predictor de los problemas de comprensión lectora. Al parecer la memoria de trabajo, específicamente su componente verbal, constituye un predictor significativo de las habilidades de decodificación y comprensión lectora. Cain, Bryant y Oakhaill (2004) demostraron que la memoria de trabajo verbal evaluada a los cinco años de edad, constituye la única de las funciones ejecutivas que predice significativamente la competencia lectora en años posteriores.

En Argentina e Italia, Carretti, Borella, Cornoldi y De Beni (2009), realizaron un estudio con niños de 8 a 14 años de edad- y con adultos jóvenes de 18 a 30 años con dificultades de comprensión. Trabajaron de modo separado con estudiantes que comprenden bien y estudiantes que tienen dificultades de comprensión, dividiendo al grupo a partir del rendimiento alcanzado en una tarea de comprensión lectora. Para medir la memoria de trabajo-, emplearon dos tipos de tareas. Los resultados confirmaron que las tareas de memoria de trabajo que implican altas demandas en términos de recursos atencionales, son mejores predictores del rendimiento en la comprensión de textos, que las tareas de memoria en la modalidad simple. En el mencionado estudio, los estudiantes con dificultades de comprensión tuvieron mayor desventaja en las tareas de la modalidad compleja cuando estas involucraban material verbal, comparados con los estudiantes que comprenden bien. De modo general, esta investigación concluyó que las dificultades comprensión lectora pueden ser atribuidas a ineficiencias en el control de los mecanismos de la memoria de trabajo.

De igual manera, son numerosos los trabajos que abordan la relación entre fluidez lectora y comprensión. La fluidez se refiere al control que alcanza el lector sobre el nivel superficial del texto, en términos de velocidad, precisión y expresividad, lo cual permite el paso a niveles profundos de procesamiento (National Reading Panel, 2000). Numerosos autores consideran que la fluidez es un componente necesario -aunque no suficiente- para comprender un texto eficientemente (Meisinger, Bloom y Hynd, 2010; Sporer, Brunstein, y Kieschke, 2009 y Yeo, 2008).

Meisinger et al., (2010) estudiaron la relación entre la comprensión lectora y la fluidez en 50 niños de 8 a 12 años de edad. Los resultados demostraron que los lectores fluidos tuvieron una comprensión normal, y los niños con distintos déficits en la fluidez lectora obtuvieron puntuaciones por debajo de la media en el test de comprensión. De esta manera mientras más amplias eran las debilidades en la fluidez, mucho más afectada se veía la comprensión. 
Otro estudio similar se realizó para identificar si había estudiantes que leían fluidamente pero no entendían el texto. Fue desarrollado por Hamilton y Shinn (2003). En el mismo participaron 66 estudiantes de tercer grado y 29 profesores de 25 escuelas primarias de los Estados Unidos. Los profesores debían dividir a los estudiantes en dos grupos: niños que podían leer palabras, pero que no comprendían lo que leían, y niños que leían con fluidez y a su vez entendían lo que leían. Los resultados revelaron que el segundo grupo obtuvo puntuaciones significativamente más altas en los test de comprensión lectora. Además, hubo una diferencia significativa en las medidas de lectura fluida entre ambos grupos. Fueron, igualmente, los escolares del segundo grupo quienes pudieron leer la mayor cantidad de palabras correctas en un minuto. Los autores concluyeron, no obstante, que es posible que sujetos que pueden leer fluidamente, no comprendan lo que leen.

En relación con el vocabulario existe, en general, un relativo consenso en cuanto a la idea de que mientras más rico resulte este, mejor será la comprensión de textos (Lubliner y Smetana, 2005). El vocabulario tiene que ver con la amplitud y diversidad léxica que se posee en relación con un idioma determinado. Se refiere a la habilidad para comprender términos y emplearlos para adquirir y transmitir significado (Hansen, 2009).

Al parecer, el vocabulario es uno de los mejores predictores para un desempeño adecuado en comprensión lectora, pues no conocer el uso o sentido de determinadas palabras se convierte en un obstáculo importante para el logro de una representación integrada que dé cuenta de la comprensión (Cromley, Snyder-Hogan y Luciw-Dubois, 2014). Saber los significados de las palabras posibilita crear las conexiones necesarias entre las ideas que provienen del texto y relacionar dichas ideas con la información almacenada en memoria.

Nation, Snowling y Clarke (2007) estudiaron la relación entre el vocabulario y la comprensión en una muestra de 24 niños de 8 a 9 años de edad. Del total de sujetos, 12 presentaban dificultades de comprensión lectora y otros tantos pertenecían al grupo control. Se les entrenó a los escolares para que asociaran nuevas formas fonológicas a las imágenes de objetos también nuevos para ellos. Los niños con dificultades de comprensión necesitaron aproximadamente la misma cantidad de intentos que los del grupo control para aprender los nuevos términos. Sin embargo, su conocimiento del significado de las palabras nuevas resultó relativamente débil y no se observó su adecuada consolidación en el tiempo. De estos hallazgos puede inferirse que la base de las dificultades para el aprendizaje del vocabulario que presentan los sujetos con un pobre rendimiento en comprensión, se 
relaciona con el componente semántico de dicho aprendizaje, más que con el fonológico. Los autores se refieren en el artículo, además, a la existencia de al menos tres posibles interpretaciones acerca de la estrecha relación entre vocabulario y comprensión. Primero, la extensión del vocabulario de un niño puede limitar la comprensión si el texto que intentan leer contiene palabras que ellos aún no conocen. En segundo lugar, mientras la lectura provee una oportunidad para aprender nuevas palabras, la comprensión puede poner un límite en el desarrollo del vocabulario. Una tercera posibilidad es que la relación entre ambas variables puede estar mediada por procesos compartidos, como por ejemplo el uso del contexto.

Cain y Oakhill (2006), realizaron un estudio longitudinal con niños de 7 a 11 años de edad para explorar el deterioro de habilidades en una muestra de estudiantes con dificultades de comprensión, en aras de identificar cualquier debilidad que pudiera estar asociada a una pobre comprensión de textos y a un inadecuado desarrollo de la lectura. Sus resultados principales revelan que los estudiantes que comprenden bien obtienen puntuaciones significativamente altas en las pruebas de vocabulario; mientras que los niños con un pobre vocabulario a los 7 años de edad, presentan dificultades en la comprensión lectora a los 11 años, lo cual demuestra que el vocabulario puede resultar un buen predictor del desempeño lector. De manera general, un pobre vocabulario conlleva a un impedimento del desarrollo de la habilidad de la lectura de palabras, lo cual se refleja en un pobre desarrollo de las habilidades de comprensión lectora.

A pesar de la gran cantidad de investigaciones al respecto (Cain, Bryant y Oakhill, 2004; Carretti, Borella, Cornoldi y De Beni, 2009; Meisinger, Bloom y Hynd, 2010; Nation, Snowling y Clark, 2007 y Sporer, Brunstein, y Kieschke, 2009) no existen resultados del todo concluyentes en cuanto a la naturaleza de la relación entre las variables comprensión lectora con la memoria de trabajo, la fluidez y el vocabulario. Tampoco se ha podido establecer con certeza la dirección o causalidad del vínculo entre vocabulario y comprensión (Cain y Oakhill, 2006 y Cromley et al., 2014). Ello se debe, en buena medida, a la heterogeneidad de las muestras de sujetos que han participado en los estudios que abordan el tema, así como a las medidas empleadas en ellos, lo cual hace difícil generalizar los resultados con un alto grado de confianza. Siendo así, el presente estudio se propone determinar la influencia que tienen en la comprensión lectora, la memoria de trabajo verbal, la fluidez y el vocabulario de un grupo de escolares cubanos de 3 ro y 5 to grados. 


\section{Metodología}

\subsection{Participantes}

Participaron en el estudio 108 niños ( $N=108)$ de 3 escuelas de Ciudad Escolar Libertad en el municipio Marianao, en La Habana. Del total, 49 niños (45\%) son de tercer grado y 59 (55\%) de quinto grado. De los escolares de tercer grado, 22 son niñas y 27 varones, con una media de edad de 8,53 años. Por su parte, de los escolares de quinto grado, 32 son niñas y 27 varones, con una media de edad de 10,58 años. Todos y todas se encontraban entre el 25 y el 95 percentil en el Test de Matrices Progresivas Coloreadas de Raven (Raven, Court, y Raven, 1986) y no presentaron ningún signo de Dislexia según el MINI BTL (Reigosa, Pérez-Abalo, Manzano, Antelo, 1994).

\subsection{Instrumentos}

En este epígrafe describimos los test usados en la investigación. El primero fue aplicado de manera colectiva, y los otros tres fueron administrados de forma individual.

Test Leer para Comprender (TLC) (Ferreres, Abusamra, Casajús, Cartoceti, Squillace y Sampedro, 2010): Consta de dos textos (narrativo e informativo) con 10 preguntas sobre cada uno, permite identificar si las dificultades de los estudiantes tienen que ver con el reconocimiento de personajes, lugar, tiempo, hechos, secuencias, cohesión y/o generación de inferencias. Cada respuesta correcta tiene el valor de 1 punto, para una puntuación máxima de 10 puntos en cada texto. Los niños que tienen dificultades de comprensión se ubican en los siguientes rangos: de 0 a 3,5 (Rendimiento Muy débil) y de 4 a 5,5 (Rendimiento Débil). Los niños que no tienen dificultades de comprensión se ubican en los rangos: de 6 a 7,5 (Rendimiento Suficiente) y de 8 a 10 (Rendimiento Óptimo).

Tarea de Memoria de Trabajo Verbal (Mclnerney, Hramok y Kerns, 2005): Se presentaron, mediante un ordenador portátil, series de 2 a 7 palabras en modalidad auditiva, a razón de una palabra por segundo. El niño debía memorizar y repetir las palabras inmediatamente después de terminada la presentación, ordenándolas de acuerdo con el tamaño real de los objetos que estas denominan, desde el más pequeño hasta el más grande. Si bien esta tarea presenta un componente espacial, pues involucra comparación de imágenes mentales, el material a recordar es verbal, por lo cual el almacenamiento y la recuperación de la información se realizan en el almacén verbal. Se presentó un bloque de entrenamiento con 4 ensayos, seguido de 12 ensayos en total, con 2 para cada cantidad de elementos $(2,3,4,5,6,7)$. Se otorgó 1 punto por cada dos objetos ordenados correctamente 
de acuerdo con su tamaño. Se recogió la puntuación total y se dividió entre la cantidad máxima posible de puntos que se puede obtener en la prueba.

Test de Fluidez Lectora: Constituye una adaptación del propuesto por Mosquera (2011). Se presentó a los estudiantes un texto de 150 palabras, evaluado por el Índice FleshSzigriszt como Muy fácil. Este se les administró a los estudiantes en una fuente Arial 12, sobre un fondo blanco, centrado en la pantalla de una computadora. Se registraba el tiempo total de lectura (en segundos) y el número de palabras del texto que lograba leer sin cometer errores. Luego se calculó una medida de fluidez lectora multiplicando la cantidad de palabras leídas por 60 y dividiendo el resultado entre el tiempo de lectura.

Prueba de Vocabulario en Imágenes de Peabody: Se empleó una versión adaptada al contexto cubano por Manzano, Piñeiro, Inguanzo, Reigosa y Morales (2000). La prueba evalúa el vocabulario receptivo. Consta de 150 placas que contienen cada una cuatro láminas (animales, acciones humanas, plantas, etc.) y una lista de 150 palabras. El niño debía seleccionar para cada palabra la lámina de la placa que más se le parezca. Cada niño obtuvo un $\mathrm{Cl}$ verbal que se deriva del percentil de vocabulario. Si ese $\mathrm{Cl}$ es menor que 75 se considera que el rendimiento del sujeto es Muy Bajo. Entre 70 y 85 las puntuaciones son Moderadamente Bajas, e identifican a sujetos que presentan dificultades en el vocabulario. Entre 86 y 115 se consideran Normales. Entre 116 y 130 son Moderadamente Altas y apuntan a sujetos sin dificultades en el vocabulario- mientras que por encima de 130 son puntuaciones Muy Altas.

\subsection{Procedimientos para analizar los datos}

Para el análisis de los datos se utilizó el software SPSS Statistics v. 18. 0. Fue empleada la estadística descriptiva para hallar medias, frecuencias y desviaciones estándar de las variables memoria de trabajo, fluidez lectora, vocabulario, y comprensión lectora. Se utilizó la t de Student para determinar la diferencia de medias de las variables estudiadas entre los estudiantes con y sin dificultades de comprensión. Se realizó un Análisis Correlacional básico (coeficiente de correlación de Pearson), para determinar la relación entre las variables. 


\section{Resultados y discusión}

\subsection{Caracterización de la comprensión lectora}

Se identificó que, en tercer grado, 30 de los estudiantes presentan dificultades para comprender textos (para un $61 \%$ de la muestra). Mientras que de los estudiantes de quinto grado, solo 16 estudiantes tienen dificultades de comprensión (para un 27. 1\%). Es importante analizar que aunque se observa un decrecimiento de la prevalencia de las dificultades de comprensión a medida que aumenta la escolarización (al comparar los resultados para tercer y quinto grados), el porcentaje de estudiantes de quinto grado que presentan dificultades para comprender textos, es mayor que el reportado por diversos autores a nivel internacional como Vellutino, Fletcher, Snowling y Scanlon (2004), quienes afirman en su investigación que la prevalencia de los trastornos de lectura en la población infantil oscila entre el $10 \%$ y el $15 \%$.

Los escolares de la muestra que presentan dificultades de comprensión, se caracterizan por tener aproximadamente 5 errores en las preguntas del test Leer para Comprender (TLC), lo cual los ubica por debajo de la media. En la mayoría de los casos, no se encontraron desventajas socioculturales que pudiesen explicar las dificultades halladas, ni tampoco déficits sensoriales.

La diferencia de medias en el puntaje de comprensión entre los grupos de tercero y quinto grados, calculado a partir de la $t$ de Student, arrojó que los estudiantes de quinto grado obtuvieron puntuaciones significativamente superiores $(p<0,001)$ que los de tercero. Cain, Lemmon y Oakhill (2004), también encontraron niños con dificultades de comprensión en tercer grado. La diferencia significativa entre ambos grados se debe, según Canet-Juric, Burin, Andrés y Ané (2007), a que es en tercer grado donde la decodificación termina de consolidarse. A partir de esta etapa los escolares transitan del aprender a leer al leer para comprender. Los niños alrededor de los siete años de edad logran el dominio de la lectura de palabras. Una vez adquirida la habilidad de decodificación o identificación de las palabras, los escolares ya están preparados para aprender nuevas habilidades relacionadas con la extracción de significados de los textos.

De manera general, los estudiantes de la muestra, con independencia del grado que cursaban, presentaron mayores dificultades $(p<0,001)$ en el texto narrativo que en el informativo. Lo anterior, discrepa con la bibliografía consultada, ya que hay autores, como McNamara y Magliano (2009)- que comparten la idea de que los textos informativos suponen mayores retos para ser comprendidos que los narrativos. Los primeros, por lo general 
presentan información nueva- y debido a la entrada de información desconocida para el lector, el procesamiento tiende a ser más lento y con más errores en la lectura. Probablemente, este resultado aflore en investigaciones con muestras más amplias. En el presente estudio, un factor que puede explicar la discrepancia citada, es la escasa familiaridad de los estudiantes con el tema abordado en el texto narrativo, una adaptación del cuento "El rebelde" de Ricardo Mariño (Ferreres et al., 2010), historia en la que se hace referencia a una familia que elige parejas para sus miembros, relacionando los nombres de los posibles cónyuges con las profesiones de estos.

\subsection{Comprensión lectora y memoria de trabajo verbal}

De los escolares de tercer grado con dificultades en comprensión lectora, 12 niños (para un 40\%) muestran problemas en la memoria de trabajo, y -se ubican en los rangos inferiores, recordando un promedio de 13 palabras de 42- y muestra problemas con el procesamiento de la información. De los escolares de quinto grado con dificultades de comprensión, 4 niños (para un 24\%) presentan problemas en la memoria de trabajo y se ubican en los rangos inferiores.

El 58\% de los estudiantes de tercer grado que no tienen dificultades de comprensión se ubican en los rangos superiores, ellos no presentan problemas con el procesamiento y recuerdo de palabras. En quinto grado -esta cifra aumenta al 67\%- y los escolares recuerdan como promedio 26 palabras.

El análisis estadístico indica que la diferencia entre tercer y quinto grados en cuanto al índice de memoria de trabajo verbal, es significativa $(p<0,001)$. Los escolares de quinto grado obtienen índices significativamente mayores, lo cual supone un mayor porcentaje de palabras recordadas durante la tarea- y una mejor ejecución en la misma. En cuanto a los escolares con y sin dificultades en comprensión, de ambos grados, también hubo una diferencia significativa $(p<0,001)$, ya que los escolares sin dificultades en comprensión obtuvieron índices superiores, lo cual significa que recuerdan una mayor cantidad de palabras y son capaces de realizar tareas complejas como la comparación de varias imágenes mentales.

La comprensión correlacionó positiva y significativamente con la memoria de trabajo verbal $(p<0,01)$ en el grupo de escolares de quinto grado. Esto coincide con lo encontrado recientemente por García-Madruga, López-Escribano, Elosúa y Gómez-Veiga (2013), 
quienes afirman que la memoria de trabajo verbal influye en la comprensión de textos, y que, por tanto, una afectación en una de estas variables traería una afectación en la otra.

\subsection{Comprensión lectora y fluidez}

De los escolares de tercer grado que tienen dificultades de comprensión lectora, 25 niños obtuvieron índices de fluidez bajos (3 niños en el rango de 0 a 35, y 22 niños en el rango de 36 a 70). Estas dificultades en fluidez se caracterizan por una lectura lenta, fragmentada y por cometer 16 errores como promedio. Los errores más frecuentes son: omisión de palabras, cambios de letras, o cambios de palabras. De los escolares de quinto grado con dificultades hay 6 que presentan problemas de fluidez, ubicándose en el rango de 36 a 70. Estos estudiantes muestran ansiedad por leer rápido al saber que se les mide el tiempo, lo que los hace equivocarse y cometer tantos errores como los estudiantes de tercer grado que presentan dificultades en este sentido.

De los escolares de tercer grado sin dificultades en comprensión, 9 se ubican en el índice de 36 a 70, lo cual supone que presentan dificultades en fluidez. No obstante, un grupo de 10 de estos estudiantes sin dificultades en comprensión, obtuvo índices medios de fluidez- de 71 a 105 y de 106 a 140. Este índice da cuenta de una lectura más veloz y precisa, con un promedio de 61 palabras leídas por minuto, y 8 errores. Los errores más frecuentes están relacionados con la omisión de letras o palabras y/o con los cambios de una letra por otra, y son propios de la recién consolidada decodificación (Canet-Juric et al., 2007). Estos escolares leen un promedio de 61 palabras por minuto. De manera general, los escolares de quinto grado obtuvieron índices mayores, lo cual supone una lectura rápida, con una mayor cantidad de palabras leídas por minuto, y menor cantidad de errores al leer.

El análisis estadístico ( $t$ de Student) indica que la diferencia de medias entre tercero y quinto grados en cuanto al índice de fluidez es significativa $(p<0,001)$. Los escolares de quinto grado obtuvieron índices mayores en fluidez. En cuanto a los escolares con y sin dificultades en comprensión, también hubo una diferencia significativa $(p<0,001)$, ya que los escolares sin dificultades en comprensión obtuvieron índices superiores. Este último resultado da cuenta de la influencia que puede tener la fluidez para la ocurrencia de dificultades de comprensión, y de la relación directamente proporcional entre estas variables.

El coeficiente de correlación de Pearson muestra una relación entre la fluidez y la comprensión para toda la muestra $(p<0,001)$, los estudiantes que presentan dificultades de fluidez por lo general presentan también dificultades de comprensión. Este resultado coincide 
con lo encontrado por Stuebing, Barth, Molfese, Weiss, y Fletcher, (2009), quienes reportan que los lectores de 8 años de edad que leían con fluidez también obtenían altos puntajes en las pruebas que evaluaban la comprensión de textos. La comprensión también correlacionó con la fluidez en los escolares de quinto grado $(p<0,01)$, lo que significa que para comprender bien el lector debe ser capaz de identificar las palabras fácil, rápidamente y sin errores Los autores también hallaron que cuanto más amplias eran las debilidades en la lectura y en la fluidez, más afectada se encontraba la comprensión.

En los estudiantes de tercer grado la comprensión no correlacionó con la fluidez, lo cual puede ser explicado porque estos estudiantes aún se encuentran entrenando el proceso de lectura y no han adquirido explícitamente a través del currículo escolar habilidades de comprensión lectora. En este grupo de niños hay mayor variabilidad.

Los resultados obtenidos en esta investigación dan cuenta de que un bajo rendimiento en la fluidez lectora está asociado a dificultades de comprensión, lo cual supone que la fluidez lectora puede ser un predictor de una buena comprensión de textos.

\subsection{Comprensión lectora y vocabulario}

De los escolares de tercer grado con dificultades en comprensión, 13 obtuvieron puntuaciones de vocabulario muy bajas o moderadamente bajas, ubicándose en los rangos de 60 a 75 y de 76 a 85 . Este grupo solía responder con más rapidez, no analizaban las opciones antes de decidir su respuesta y se distraían fácilmente. El tiempo de ejecución de la tarea fue menor, debido a sus limitaciones de vocabulario. Pocos de estos escolares se interesaban en buscar, posteriormente, las palabras que desconocían en el diccionario. La media del $\mathrm{Cl}$ verbal de este grupo fue de 96,67 (ds=14,84). Estos escolares tienen un retraso del lenguaje de 1,68 años, y su edad promedio de vocabulario es de 7,56, mientras que la media de edad cronológica fue de 8,52, presentando discrepancias entre ambas edades (cronológica y de vocabulario).

En cuanto a los escolares de quinto grado con dificultades en comprensión lectora, 6 obtuvieron puntajes muy bajos o moderadamente bajos, 8 se consideran normales y sólo 2 alcanzaron puntajes moderadamente altos. Los datos reportan que la media de $\mathrm{Cl}$ de vocabulario de este grupo es de 94,75 (ds=12,61), dato similar al promedio de $\mathrm{Cl}$ de vocabulario de los escolares con dificultades en comprensión de tercer grado. La media de edad de vocabulario es de 8,88 años, y su edad real promedio de 10,57. El retraso en el lenguaje es de 2,06 años. El comportamiento de estos niños durante la prueba de 
vocabulario fue similar al de los escolares de tercer grado, con excepción de la rapidez al contestar. Ellos solían mirar bien cada una de las opciones antes de responder. No obstante, al tener un vocabulario muy limitado sus respuestas muchas veces eran incorrectas. Una diferencia que se pudo apreciar en los niños con dificultades en comprensión de tercer y quinto grados fue que estos últimos se interesaban por conocer el significado de las palabras que no conocían y al terminar el test buscaban fuentes externas como la maestra, el diccionario, y otros niños para lograrlo.

Los resultados encontrados en ambos grados para los niños con dificultades de comprensión coinciden con los hallazgos de Nation, Snowling y Clark (2007), quienes describieron un vocabulario más débil en niños con problemas para comprender los textos, quienes desconocían muchas palabras lo cual obstaculizaba la interpretación de frases y la generación de inferencias.

Por su parte, de los escolares de tercer grado que no tienen dificultades en comprensión lectora, 6 obtuvieron puntajes muy bajos y moderadamente bajos en la prueba de vocabulario, ubicándose en los rangos de 60 a 75 y de 76 a 85 . Mientras que 5 se ubican en los índices que representan un vocabulario normal, y 8 obtuvieron puntajes moderadamente altos o muy altos, situándose en los rangos superiores. La media de $\mathrm{Cl}$ verbal de este grupo es de 104 (ds=15,81). Su promedio de edad de vocabulario es 8,42 años, y el promedio de edad real 8,55 años. Puede apreciarse la similitud entre ambas edades, lo que indica un desarrollo adecuado del vocabulario. Su tiempo de ejecución de la prueba solía ser mayor, debido a que conocían más palabras que el grupo de niños con dificultades en comprensión.

De los escolares de quinto grado sin dificultades en comprensión, 11 obtuvieron puntajes moderadamente bajos o muy bajos, mientras que 17 se consideran normales y 15 alcanzaron los rangos superiores que pertenecen a los puntajes moderadamente altos o muy altos. Este grupo presenta un Cl de vocabulario promedio de 103,56 (ds=15,33), media similar a los de los niños sin dificultades en comprensión de tercer grado. La edad promedio de vocabulario de estos niños es de 10,24 años, mientras que la media de su edad real es de 10,59. El retraso del lenguaje en este grupo es de 1,29 años como promedio, 28 niños (47\%) presentan retraso y 31 (53\%) no tienen retraso e incluso mostraron niveles de vocabulario superiores a su edad real. Dos de ellos llegaron hasta el final de la prueba, lo cual indica su alto nivel de desarrollo en este sentido. 
El análisis estadístico usando la $t$ de Student indica que la diferencia entre ambos grados en cuanto al $\mathrm{Cl}$ de vocabulario no es significativa. Sin embargo, entre los escolares con y sin dificultades en comprensión hubo una diferencia significativa $(p<0,01)$. Los estudiantes sin dificultades en comprensión obtuvieron un $\mathrm{Cl}$ verbal superior, lo cual da cuenta de una edad de vocabulario acorde a su edad cronológica y un mayor dominio del significado de las palabras. Este resultado corrobora nuevamente lo encontrado por Nation, Snowling y Clark (2007), quienes apuntaron que la comprensión y el vocabulario eran variables directamente proporcionales e interdependientes. Los resultados obtenidos en esta oportunidad también coinciden con Snowling y Hulme (2011), quienes encontraron que los escolares con dificultades en comprensión lectora también muestran dificultades en el vocabulario receptivo.

El coeficiente de correlación de Pearson muestra una correlación positiva entre ambas variables (comprensión y vocabulario) para el grupo de quinto grado $(p<0,05)$, lo que significa que el nivel de vocabulario influye en la comprensión de textos. Mosquera (2011) concuerda en que el tener un pobre vocabulario se convierte en un obstáculo para el logro de la decodificación rápida y eficaz, y por tanto para lograr la comprensión. En este sentido, el conocimiento previo de los sujetos, la práctica de la lectura y la profundización en diferentes temas, aumentan el nivel de vocabulario y por lo tanto favorecen una mejor comprensión de textos.

Los resultados de la presente investigación permiten afirmar que, para este grupo de estudio, la comprensión y el vocabulario tienen una relación de interdependencia, lo cual coincide con lo encontrado por autores como Nation, Cocksey, Taylor y Bishop (2010), y Cain y Oakhill (2006). Esto significa que aquellos niños que presenten un deficiente desarrollo del vocabulario, presentarán, así mismo, dificultades de comprensión, y viceversa.

\section{A modo de conclusiones}

La comprensión lectora es un proceso complejo del cual participan otros procesos cognitivos, que condicionan la eficiencia del acto de comprender un texto. No es de extrañar que el perfil cognitivo de los sujetos que presentan dificultades de comprensión, pueda resultar heterogéneo. Es este estudio, no obstante, se comprobó la influencia de la memoria de trabajo verbal, la fluidez lectora y el vocabulario en el desempeño en comprensión de un grupo de estudiantes de nivel primario de educación. 
Específicamente, se aprecia que- para esta muestra- la memoria de trabajo verbal explica la variabilidad en el rendimiento en comprensión lectora, sobre todo, para los estudiantes de quinto grado. Lo mismo sucede con el vocabulario y la fluidez es otro factor que explica esta variabilidad tanto en tercero como en quinto grados.

Lo anterior tiene sentido si se toma en cuenta la etapa del desarrollo por la cual transitan los estudiantes que componen la muestra. En tercer grado, los procesos de decodificación (asociados a la fluidez lectora) no se han consolidado, por lo cual estos procesos más básicos determinan en gran medida, en esta edad, el acceso al significado del texto que se lee.

Por otra parte, el hecho de que se hayan encontrado un mayor número de estudiantes con dificultades de comprensión en tercer grado, respecto a los de quinto, habla también a favor de la influencia de la escolarización sobre el proceso de comprensión de textos.

\section{Referencias}

Abusamra, Valeria, Ferreres, Aldo, Raiter, Andrea, De Beni, Rosana y Cornoldi, César. (2010). Test Leer para Comprender TLC. Evaluación de la comprensión de textos. Buenos Aires: Paidós.

Baddeley, Allan David y Hitch, Graham. (1974). Working memory. En Gordon Bower (Ed.), The psychology of learning and motivation: Advances in research and theory (pp. 4789). New York: Academic Press.

Cain, Kate, Bryant, Peter y Oakhill, Jane. (2004). Children's reading comprehension ability: Concurrent prediction by working memory, verbal ability, and component skills. Journal of Educational Psychology, 96(1), 31- 42.

Cain, Kate, Lemmon, Kate y Oakhill, Jane. (2004). Individual differences in the inference of word meanings from context: the influence of reading comprehension, vocabulary knowledge, and memory capacity. Journal of Educational Psychology, 96(4), 671-681.

Cain, Kate y Oakhill, Jane. (2006). Profiles of children with specific reading comprehension difficulties. British Journal of Educational Psychology, 76, 683-696.

Canet-Juric, Lorena, Burin, Débora Inés, Andrés, María y Ané, Alejandra. (2007). Medición de inferencias en la comprensión lectora. En Mirta Ison y María Cristina Richaud (Eds.), Avances en investigación en ciencias del Comportamiento en Argentina (pp. 815-829). Universidad del Aconcagua. Mendoza, Argentina.

Carretti, Bárbara, Borella, Erika, Cornoldi, César y De Beni, Rossana. (2009). Role of working memory in explaining the performance of individuals with specific reading comprehension difficulties: A meta-analysis. Learning and Individual Differences, 19, 246-251. 
Catts, Hugh, Adlof, Suzanne y Ellis-Weismer, Susan. (2006). Language deficits in poor comprehenders: A case for the simple view of reading. Journal of Speech-LanguageHearing Research, 49, 278-293.

Cromley, Jennifer, Snyder-Hogan, Lindsey and Luciw-Dubois, Ulana (2014). Cognitive activities in complex science text and diagrams. Contemporary Educational Psychology, 35(1), 59-74.

Demagistri, María Silvina, Canet, Lorena, Naveira, Liliana y Richard, María. (2012). Memoria de trabajo, mecanismos inhibitorios y rendimiento lecto-comprensivo en grupos de comprendedores de Secundaria Básica. Revista Chilena de Neuropsicología, 7(2), 72 78.

Ferreres, Aldo, Abusamra, Valeria, Casajús, Andrea, Cartoceti, Romina, Squillace, Mario y Sampedro, Bárbara. (2010). Pruebas de screening para la evaluación de la comprensión de textos. Revista Neuropsicología Latinoamericana, 1, 41-56.

García-Madruga, Juan Antonio, López-Escribano, Carmen, Elosúa de Juan, María Rosa y Gómez-Veiga, Isabel. (2013). A predictive study of reading comprehension in thirdgrade Spanish students. Psicothema, 25(2), 199-205.

González, Klency. (2008). Propuesta de un programa para mejorar la comprensión de textos en estudiantes universitarios. Revista Actualidades Investigativas en Educación, 8(2), $1-31$.

Hamilton, Chad y Shinn, Mark. (2003). Characteristics of Word callers: An investigation of the accuracy of teachers' judgments of reading comprehension and oral Reading skills. School Psychology Review, 32, 228-240.

Kintsch, Walter y Van Dijk, Teun. (1978). Toward a model of text comprehension and production. Psychological Review, 85(5), 363- 394.

Kintsch, Walter. (1998). Comprehension, a paradigm for cognition. New York, Cambridge University Press.

Lubliner, Shira y Smetana, Linda. (2005). The Effects of Comprehensive Vocabulary Instruction on Title I Students' Metacognitive Word-Learning Skills and Reading Comprehension. Journal of Literacy Research, 37(2), 163-200.

Manzano, Mayra, Piñeiro, Armando, Inguanzo, Gonzalo, Reigosa, Vivian y Morales, Aymara. (2000). Adaptación y normación de la prueba de vocabulario Peabody en la población cubana. Revista Cubana de Psicología, 17(2), 35-47.

Martínez, Tomás, Vidal-Abarca, Eduardo, Sellés, Pilar y Gilabert, Ramiro. (2008). Evaluación de las estrategias y procesos de comprensión: El Test de Procesos de Comprensión. Infancia y Aprendizaje, 31(3), 319- 332.

McNamara, Danielle y Magliano Joe. (2009). Towards a Comprehensive Model of Comprehension. Psychology of Learning and Motivation, 51. Advance online publication. doi: 10.1016/S0079-7421(09)51009-2. 
McInerney Robert, Hrabok Marianne and Kerns, Kimberly. (2005). The children's sizeordering task: A new measure of nonverbal working memory. Journal of Clinical and Experimental Neuropsychology, 27, 735-745.

Meisinger, Elizabeth, Bloom, Juliana y Hynd, George (2010). Reading fluency: implications for the assessment of children with reading disabilities. Ann. of Dyslexia, 60, 1-17.

Mosquera, Raysil. (2011). Valor predictivo del procesamiento de la palabra escrita en la competencia lectora en el idioma español. (Tesis en opción al título académico de Máster en Neurociencias). Centro de Neurociencias de Cuba, La Habana, Cuba.

Nation, Kate, Snowling, Margaret y Clarke, Paula. (2007). Dissecting the relationship between language skills and learning to read: Semantic and phonological contributions to new vocabulary learning in children with poor reading comprehension. International Journal of Speech-Language Pathology, 9(2), 131-139.

Nation, Kate, Cocksey, Joanne, Taylor, Jo y Bishop, Dorothy. (2010). A longitudinal investigation of early reading and language skills in children with poor reading comprehension. Journal of Child Psychology \& Psychiatry, 51, 1031- 1039.

National Reading Panel. (2000). Report of the National Reading Panel. Teaching children to read: An evidence-based assessment of the scientific research literature on reading and its implications for reading instruction: Reports of the subgroups (NIH Publication No. 00-4754). Washington, DC: U.S. Government Printing Office. Recuperado de https://www.nichd.nih.gov/publications/pubs/nrp/Documents/report.pdf

Perfetti, Charles, Landi, Nicole, and Oakhill, Jane. (2005). The acquisition of reading comprehension skill. En Margaret Snowling y Charles Hulme (Eds.), The science of reading: A handbook (pp. 227-247). Oxford: Blackwell.

Raven, John C., Court, John Hugh y Raven, John. (1986). Manual for Raven"s Progressive Matrices and Vocabulary Scales (Section 2) - Coloured Progressive Matrices (edition with U.S. norms). London: Lewis.

Reigosa, Vivian, Pérez-Ábalo María C., Manzano, Mayra y Antelo, José M. (1994). Sistema automatizado para explorar la lectura en escolares de habla hispana. Revista Latina de Pensamiento y Lenguaje, 2(1), 141-159.

Ricketts, Jesse, Nation, Kate, and Bishop, Dorothy. (2007). Vocabulary is important for some, but not all reading skills. United Kingdom: University of Oxford.

Snowling, Margaret y Hulme, Charles. (2011). Evidence-based interventions for reading and language difficulties: Creating a virtuous circle. British Journal of Educational Psychology 81, 1-23. Recuperado http://onlinelibrary.wiley.com/doi/10.1111/bjep.2011.81.issue-1/issuetoc

Sporer, Nadine, Brunstein, Joachim y Kieschke, Ulf. (2009). Improving students' reading comprehension skills: Effects of strategy instruction and reciprocal teaching. Learning and Instruction, 19, 272-286. 
Stuebing, Karla, Barth, Amy, Molfese, Peter, Weiss, Brandon y Fletcher, Jack. (2009). IQ Is Not Strongly Related to Response to Reading Instruction: A Meta-Analytic Interpretation. Except Child, 76, 31-51.

Swanson, H. Lee y Beebe-Frankenberger, Margaret. (2004). The relationship between Working Memory and Mathematical Problem Solving in children at risk and not at risk for serious Math difficulties. Journal of Educational Psychology, 96(3), 471- 491.

Vellutino, Frank, Fletcher, Jack, Snowling, Margaret y Scanlon, Donna. (2004). Specific reading disability (dyslexia): what have we learned in the past four decades? Journal of Child Psychology and Psychiatry, 45(1), 2-40.

Yeo, Seungsoo. (2008). Relation between 1- Minute CBM Reading Aloud Measure and Reading. Minnesota University, United States of America. 